\title{
Nuevos pasos en el desencantamiento: La sociología del currículum
}

\section{Isidoro Alonso}

"Todo orden colectivo está siempre legitimado por definiciones oficiales; descubrir que eso no es mas que parte de la realidad, o peor asin, que sirve para ocultar lo que realmente pasa, es intrinsicamente subversivo para el "buen orden" oficial... En pocas palabras, que el mundo no es 10 que pareces. Peter Berger. "Sociology Reinterpretred", päg. I2.

\section{SOCIOLOGIA DEL CURRICULUM, ¿A CUENTO DE QUE?}

¿Qué pinta un sociólogo, es posible que se pregunte alguien, metido en temas curriculares? ¿No es éste un campo técnicamente bien delimitado y con sus propios especialistas? ¿No estaremos ante un asalto o invasión más a la intimidad y lo reservado, en los que la sociología parece acompañar a la información? Trataré de mostrar, aunque sea esquemáticamente, que efectivamente la sociologia tiene mucho que decir en este tema; otra cosa es que lo diga o no y que lo que diga tenga o no sentido. Es más, su actuación seria en este campo, como en otros, pondria en evidencia que hay más profesionales que tienen algo que decir y que lo que digan unos y otros le interesa incluso al simple ciudadano, porque de alguna manera le afecta. En cuestiones de saberes no se justifican exclusivismos; en nuestro caso las paredes del "secret garden" deben ser derribadas y licenciado el "secret service».

Respecto de la sociología misma trataré de mostrar varias cosas. Una, que este nuevo afán no es un capricho o incursión festiva de los llamados nuevos sociólogos de la educación, como con frecuencia se ha dicho; por el contrario, cuando ha habido buena sociologia ésta ha llegado hasta los contenidos de la educación, a los que ha dado la importancia que tienen. Otra, que su presencia o ausencia en el tema, no es tampoco cuestión de simple azar o de lucha por nuevas parcelas de ejercicio profesional; es más bien reclamo y urgencia puestos al descubierto por cambios de muy distinta indole. En próxima ocasión trataré de mostrar por qué entre nosotros no se ha hecho como se viene ya haciendo en otras partes y por qué incluso puede sorprender que se haga.

Para llegar donde me propongo, un camino sería precisamente mostrar cómo se hace en esos otros paises o, si se prefiere, en el ámbito internacional, donde hace tiempo que habituales prácticas corporativas pusieron cartel a la parcela: sociologia del currículum. Otro procedimiento seria ofrecer una argumentación coherente de por qué tiene que ser así. Seguiré un camino hibrido, argumentando, haciendo referencias a circunstancias y autores significativos, siguiendo una minima secuencia histórica.

Para empezar diré algo, con perdón de los especialistas, de lo que se entiende por currículum; algo también de lo que trata 
la sociologia, con el perdón de los sociologos, y algunas cosas más condensando la incidencia de ésta sobre aquél, es decir, sobre lo esencial de la llamada sociologia del curriculum.

Desgraciadamente, el "uanglicismo" de referencia no tiene un sentido univoco ni en su propio idioma de origen, por lo que no es de extrañar que no lo tenga en el nuestro, ni en el uso generalizado que de él se viene haciendo. En todo caso, el término ha experimentado, o está experimentando,..según los casos, dos procesos expansivos simultáneos: uno en ese uso casi ya universalmente aceptado, otro en su propio contenido. En este aspecto, y por hacer unas citas ilustrativas entre las más a mano, tanto se puede entender por curriculum waquellas experiencias de aprendizaje que intencionalmente han sido diseñadas por las organizaciones educativas formales». (Musgrave, pág. 41), como «todo lo que ocurre en el aula como consecuencia de lo que hacen los "profesores" (Oliver, pág. 6s), o simplemente unna elección hecha de la cultura de una determinada sociedad" (Lawton, 1981, pág. IIs).

La expansión del concepto en una y otra dirección ha ido acompañada, o mejor, ha sido producida, por los cambios educativos y sociales en general ocurridos de modo más o menos peculiar en cada país, sin olvidar los objetivos culturales y políticos predominantes en las respectivas instituciones educativas: Asi, en los Estados Unidos, ha contado la concepción de la escuela como extensión de la comunidad y como agente decisivo en la americanización de su heterogénea población; en Francia ha tenido una especial significación el objetivo «instructivo" y laico; en el Reino Unido la socialización moral y política en su versión dual, elitista y popular; entre nosotros quizá la cambiante $y$ radical alternativa sacralizante-secularizadora, en su versión clerical-anticlerical. La propia forma de gestión educativa, descentralizada de los sajones y centralizada de los latinos, ha pesado en la configuración histórica de los contenidos curriculares, su regulación e incluso en la significación y uso del término (Lauglo, 1979). Pero son, sin duda, las estructuras políticas las que más decisivamente configuran el curriculum. Un sistema autoriúnico y rígido, sino que lo empapará de su propia doctrina; un sistema democrático, es decir, pluralista y descentralizado, permitirá la incorporación de diferencias sobre una proporción mayor o menor de contenidos comunes.

De otro lado, ¿de qué se ocupa la sociologia? Es evidente que una respuesta adecuada no podria ser breve ni tampoco única, después de la "crisis de la sociología y de la crisis de la crisis", es decir, cuando hemos llegado al general reconocimiento y coexistencia de las diversas perspectivas nacidas en los sesenta y enfrentadas con tanta frecuencia. En los últimos tiempos a la coexistencia, más o menos desigual, según los casos, de las̀ posiciones normativas, la funcionalista $y$ la crítica, se han añadido los enfoques subjetivos, interpretativos e interactivos, centrados todos ellos en ámbitos pequeños y destacando el papel de los individuos en la configuración de su mundo social, en la «construcción de la realidad socialy.

Asi pues, mientras unos ven desde la sociología estructuras o sistemas, en conflicto o en consenso, otros ven o se sitúan en el lugar de los actores sociales, moviéndose más o menos condicionados en esas estructuras y en algún grado modificándolas. Condensando esas posiciones, alguien las ha caracterizado, respectivamente, por la armonia, el conflicto y las contradicciones e ironías.

El renacimiento de esa tercera perspectiva ha incidido doblemente el estudio del curriculum; de un lado, al estudiar la educación en su ámbito microsocial, del aula principalmente, $y$, de otro, al centrarse en el conocimiento y su definición social; más concretamente en el conocimiento a transmitir en la institución escolar. Asi pues, el nacimiento; si no la explosión, de la nueva sociología del curriculum es obra de los «nuevos sociólogos de la educación» surgidos de ciertas perspectivas teóricas (fenomenológica e interaccionista principalmente), de su aplicación a objetos de estudio confluyentes (la educación y el conocimiento que imparte) y en circunstancias educativas y sociales, especialmente descentralizadas y dinámicas (las del Reino Unido en el último decenio).

Asi pues, esa supuesta nueva sociología de la educación nace sustancialmente como sociología del conocimiento y pron- 
to del curriculum, manifestándose en unos documentos considerados como cruciales (Young, M. F. D., 1971). Para poner de manifiesto la rápida y fuerte incidencia de la sociologia en el curriculum me voy a permitir transcribir unas frases iniciales de esos documentos clave:

"Cómo una sociedad selecciona, clarifica, distribuye, transmite y evalúa el conocimiento educativo que considera público refleja, a la vez, la distribución del poder y los principios de control socialy (Bernstein, M., 1971, p. 47).

«Sabemos muy poco de cómo el conocimiento llega a estar organizado en las instituciones educativas. Esto es aplicable no sólo a los modos en que sus formas institucionales regulan las estructuras "válidas" de la experiencia educativa, sino mucho más a las concreciones de estas formas en la propia realidad individual de alumnos y profesores" (Esland, G. F., 1971, P. 70).

Estas "citas tan citadas" marcan un momento y una dirección más o menos nueva, pero que, en todo caso, han sido continuadas por abundantes y variados trabajos a lo largo de la década transcurrida. Tan abundantes, que la sociologia de la educación sajona es hoy, más que nada, sociologia del curriculum, y $\tan$ variados que han ido situándose tanto en la nueva sociologia como en las viejas perspectivas, hasta hacer que todos ellos se ocupen intensamente del curriculum. Y si nos atenemos a las posiciones ideológicas subyacentes, esas diversas posiciones teóricas de las sociologías de la educación, nos encontraremos, como con los sociólogos en general (Alonso Hinojal, 1980, páginas 18 y ss.), dos posiciones últimas más - menos radicales: los partidarios de la neutralidad o el "statu quo", por un lado, y los partidarios de la ruptura y el compromiso político, por orro. De ahí que al final, se hablará del inevitable paso de la sociologia a la politica del curriculum; pero antes recorramos las etapas previas.

\section{EL SUPUESTO OLVIDO DEL CONTENIDO DE LA EDUCACION}

No estoy de acuerdo con la afirmación casi ritual y que inicia otro de los artículos incluidos en la obra citada como punto de arranque del actual interés por el curriculum entre los sociólogos. No estoy de acuerdo con que «el casi total olvido por los sociólogos de cómo se selecciona, organiza y evalúa el conocimiento en las instituciones educativas apenas necesite ser fundamentado" (Young, M. F. D., 1971, p. 19). Mi punto de vista, ya adelantado, es que cuando hubo sociología de la educación, hubo sociologia del curriculum. Cuando aquélla entró en el estrecho desfiladero de una sola perspectiva y ésta acritica, es lógico que se olvidara de cuestionar aspectos fundamentales en el mantenimiento de la situación, por afectar a cuestiones de distribución de poder y mecanismos de control en la educación. Por otra parte, los autores sajones han tenido la tradicional costumbre de no considerar otra sociologia que la que se escribe en inglés y de practicar una rutina muy generalizada, cual es la de fijar la atención en unos pocos y grandes autores, cuando se busca en el pasado inspiración para el presente.

Puesto que yo sí considero necesario documentar minimamente mis afirmaciones, pasaré brevemente sobre el Durkheim sólo recientemente conocido por recientemente traducido, por el olvidado y no considerado sociólogo Thorstein Veblen, y por el Mannheim si considerado sociólogo, pero apenas incluido entre los sociólogos del curriculum. La obra de este último, además de estar mucho más próxima al actual desarrollo del tema en Gran Bretaña, es obligada aqui por partida doble: como sociólogo del conocimiento y como sociólogo de la educación y en ella del currículum. El que destaque en relación con el curriculum autores que en otra ocasión destaqué en la historia de la sociologia de la educación no es casualidad, como tampoco es simple comodidad o personal debilidad (Alonșo Hinojal, I, 1980).

Si fuera licito distinguir dos sociólogos de la educación en la obra de Durkheim, el primero, el de la educación moral, ha sido prácticamente el único tenido en cuenta al considerar el contenido de la educación y no el segundo, el autor de "L'evolution pédagogique en France». En la primera dimensión es conocida la argumentación general del autor: preocupado por la reconstrucción moral de la sociedad francesa, busca una ciencia de la moralidad y encuentra la sociologia; bus- 
ca el instrumento de moralización y encuentra la educación, como teoría, y la pedagogía como práctica. El contenido de la educación, cuya concreción encomienda a la sociologia, emana de cada sociedad, $e$ incluso de cada una de sus partes y de cada momento de su historia. Al Estado, como agencia moral fundamental, corresponde asegurar que dichos contenidos reflejen, por un lado, los valores y normas sociales básicos $y$, por otro, los adecuados a las funciones que cada ciudadano haya de desarrollar en sociedad. ¿Y cuáles son los contenidos de esa educación moral? Fundamentalmente, tres: la disciplina, en su doble vertiente, asegurando la regularidad del comportamiento social y el cumplimiento de los papeles sociales; la adhesión y fidelidad a los grupos sociales $y$ entre ellos a la sociedad política y, finalmente, la autonomia de la voluntad compatible con lo anterior gracias a la fuerza de la razón.

Pero hay que considerar, sobre todo, al «segundo Durkheim» para el tema que aquí se considera. Es el que desciende del plano doctrinal y especulativo al empiri$\mathrm{co}$, al histórico concretamente; el que muestra en ese recorrido histórico la relativa autonomia de las instituciones educativas y su capacidad de adaptación a las nuevas circunstancias; el que destaca el papel del conflicto en la dinámica educativa y da un tratamiento detenido y complejo al propio conocimiento escolar, nada ajeno a ese conflicto de intereses en la educación.

Los dos últimos capitulos de "L'education pédagogique en Francè son especialmente significativos en relación con el currículum. «El gran problema pedagógico es, pues, saber cuáles son los objetivos hacia los cuales hay que orientar la reflexión del alumno... Ahora bien, el pensamiento sólo tiene dos grandes objetivos: el hombre y la naturalezas (Durkheim, E., r9, p. 408). Pero el currículum no se ocupa sólo del qué, sino también del cómo, y del cómo se ccupa también Durkheim. Para que el niño llegue a conocer al hombre no hay otro medio que ponerle frente a él, en su doble dimensión histórica y actual. Pero por debajo de la efectividad de todo procedimiento debe estar el convencimiento de que es necesario que adquiera ese conocimiento; a quien sienta esa necesidad, «todo podrá servirle como material, a pesar de los programas». Dentro de la historia hay forzosamente que presentar la literatura $y$, junto a ella, la lengua, Mediante la literatura, el alumno podrá "tocar" los distintos pueblos ucon las manos" y "verlos vivir" en los documentos. La lengua no sólo permitirá un mejor y más fiel conocimiento de los documentos, sino también de la lógica científica con que fue elaborado el conocimiento del mundo, además de ser parte fundamental de su cultura.

El otro gran objetivo es el conocimiento de la naturaleza, cuya justificación trató de reforzar con diversas argumentaciones, desde las "utilitarias y profesionales", hasta las tipicamente humanistas (sin estos conocimientos no habrá " mmente completa»), sin olvidar las razones morales o propiamente "educativas".

Veblen es ineludible aquí por derecho propio y no por mi propio afán de incorporarlo y mantenerlo entre los clásicos de la sociologia de la educación. Después de él podrían también citarse otros sociólogos que, al paso, se fijaron en la importancia del contenido de la educación en la estructuración de la sociedad moderna desde D. Riesman en su «Multitud solitaria» hasta Whyte en «El hombre organización".

Con Veblen ocurre algo parecido a lo que ocurre con Mannheim, que tanto pueden ser considerados sociólogos del conocimiento como del curriculum. En efecto, para Veblen, los saberes son productos sociales, intercambiables con otros bienes sociales (prestigio, dinero, etc.), que gozan de alto "status" social, "status" que transmiten a sus poseedores en cuanto elementos de distinción y poder, aunque ellos mismos, los saberes están jerarquizados como jerarquizados quedan en la estructura social sus rèspectivos detentadores. El saber cientifico es todo menos estable y neutro; es decir, se orienta hacia ciertos objetivos sociales preferentemente, y en si mismo es discriminatorio, resistiendo la incorporación de saberes nuevos. (Aquí suena a Kuhn y a otros autores actuales.)

Al relacionar la estructura social con el aula y sus saberes, Veblen presta una especial atención al lenguaje, criticando agudamente lo que hoy llamamos uprivación lingüistica». Para él, todo grupo, cualquiera que sea su posición social, 
posee los recursos lingüisticos adecuados a las necesidades de su forma de vida (Veblen, T., cap. XIV).

Como obra de Mannheim más significativa para el tema en consideración me referiré a su obra póstuma «Introducción a la Sociología de la Educación", en la que se ocupa de la sociologia como materia concreta a enseñar y como enfoque específico para el estudio de la propia edacación. Es la primera vertiente, naturalmente, la pertinente aqui. En ella encontramos tres contenidos curriculares destacables: a) aspectos generales de todo currículum; b) ciertos elementos del curriculum cívico-social; c) el contenido propiamente sociológico para estudiantes de magisterio.

Sobre el curriculum en general hará Mannheim afirmaciones $\tan$ importantes y de actualidad como que tiene un significado tanto social como cultural y politico, que sus diseñadores deben tener en cuenta el qué, el para quién, el cuándo y el cómo debe ser enseñado. El conjunto, dirá, debe constituir un todo armónico como una pieza musical escrita para una gran orquesta. En la descripción negativa del curriculum incluirá el que éste sea wuna simple colección de innumerables datos", o el resultado de luchas imperialistas entre disciplinas erigidas en «estados imperiales». Por razones de brevedad y evidencia prescindo de comentarios a estas alusiones tan significativas.

Respecto del curriculum civico-social dirá también cosas tan pertinentes y hoy ya de general circulación como que debe contener tanto elementos cognoscitivos como emocionales, que su contenido y objetivos pueden ser tanto fascistas como democráticos y que debe llegar más allá de los puros conocimientos políticos para incluir las relaciónes sociales características de un sistema u otro.

En el tercer nivel y más concreto de los tres que he diferenciado, ¿qué sociologia debe conocer un candidato a profesor? Ante todo los conceptos y métodos básicos para poder acercarse personalmente al conocimiento de los fenómenos sociales. Como especificación de lo anterior, deberá conocer los procesos sociales elementales que permitan entender los grandes cambios o crisis. Progresando en concreción deberá conocer el significado de la educación en el contexto social en que se encuadra. Debe, finalmente, llegar a saber cuáles son las conexiones entre la educación escolar y la que se produce en otros ámbitos institucionales, concretamente en la familia, la comunidad y la Iglesia. En resumen, diferencia claramente algo que hoy sigue confuso para muchos metidos en estos berenjenales: la sociología para educadores, de la sociología de la educación y de la sociología de la enseñanza. Sus precisiones y recomendaciones son muy precisas y a la vez variadas, como cuando pide tratar los temas con profundidad ante el peligro de que "personas no especializadas se califiquen a si mismos sociólogos de la educación y por su diletantismo estropeen tanto la reputación de esta disciplina como la profesión de educador" (Mannheim, pág. 233).

Consecuentes con las pretensiones de este trabajo, las notas anteriores no se refieren más que a tres autores que considero significativos; había otros a considerar. De esos tres autores es claro que no he pretendido más que entresacar algunas ideas para mostrar su relevancia en el tema que se está considerando. Su estudio a fondo con esta preocupación seguro que merecería la pena.

\section{DE LA NUEVA SOCIOLOGIA DE LA EDUCACION A LA SOCIOLOGIA DEL CURRICULUM}

Al comienzo de los años setenta se produce el renacimiento de los estudios del curriculum, especialmente entre los sociólogos, renacimiento que llega envuelto en otro más amplio de los estudios sociológicos sobre la educación que algunos pronto consideraron una nueva sociología de la educación. Como dije al principio, una publicación concreta se considera la proclama de una y otra corriente y su fecha de aparición el momento de arranque. También hay unos autores a quienes se considera protagonistas destacados y no por casualidad. "La idea de este libro (dirá su compilador y primer autor) fue concebida por primera vez en una discusión entre Pietre Bourdieu, Basil Bernstein y yo mismo (Michael Young) después de la conferencia de Darham de la Asociación Británica de Sociologia en abril de 1970" (Young, M. 
[ः. D., 1971, l'retace). Como ocurre a veces con ciertas publicaciones, o hechos sociales de diversa indole, las derivaciones y consecuencias de su aparición van mucho más allá de las intenciones y previsiones de sus autores. En este caso, sus autores nunca pretendieron, como luego se ha considerado por algunos, iniciar ninguna unueva definición de la sociologia, sino abrir algunas alternativas $y$ disecciones provechosas para la investigación en sociología de la educación» y abordar uuna serie de problemas que hasta ahora parece que han sido generalmente desconsiderados".

No voy a volver aquí sobre el nacimiento de esa supuesta nueva sociología de la educación, ni hasta qué punto es nueva o vieja, puesto que lo he sintetizado en otro lugar (Alonso Hinojal, I., 1980, páginas I $1-164)$. Lo que si procede ahora es resaltar aquellos rasgos de la nueva corriente y aquellas circunstancias de su nacimiento que se refieren más directamente al curriculum, para-mostrar que la atención a éste constituyó el núcleo de aquélla. Después condensaré las ideas de los autores más significados sobre el tema.

Young pretende ante todo prestigiar la sociologia de la educación animando la discusión teórica, que él veia muy reducida o ausente, reorientándola temáticamente hacia nuevos problemas, o hacia viejos problemas pero replanteados (whechos» por el propio investigador y no simplemente "tomados" de otros o dados por supuestos). Entre los problemas a considerar el principal fue el conocimiento a transmitir, que se "tenia" como dado e incuestionado, cuando es relativo y problemático como construcción social que es. Por eso, para Young la sociologia de la educación no puede ser concebida como distinta de la sociología del conocimiento, de la que toma argumentos y conceptos criticos como el de la usubversión del absolutismo» impuesto por los conocimientos reconocidos socialmente como absolutos (Douglas), o los propios criterios de validez $y$ rigor científico (Mills), en el fondo también socialmente construidos y relativos.

Pero donde está más clara la identidad entre sociología de la educación, sociologia del curriculum y sociologia del conocimiento es en su trabajo sobre "Un como conocimiento socialmente organizadon, en cl que Young aborda succsivamente: los cambios temáticos en las discusiones sobre educación y las posibilidades y limitaciones de las sociologias de la educación y del conocimiento convencionales para el estudio realmente productivo del curriculum, para terminar sugiriendo un "marco y posibles direcciones" para el curriculum, puesto que es el tema de actualidad según él.

La aportación de Bernstein destaca por su intento de ligar la estructura del conocimiento escolar y la forma de transmitirlo con la estructura del poder y control social. El intento, sin duda demasiado ambicioso, no podía ser cubierto más que con unos instrumentos conceptuales de un grado enorme de abstracción. Son éstos los conocidos de "classification»y "framing", tan socorridos por el autor para el análisis de los temas más diversos. Aquí son aplicados a deslindar dos tipos de currículum, los «integrados» y los de tipo "colección" en razón de que las separaciones entre ramas del saber sean más o menos rigidas y mayores o menores las posibles modificaciones a introducir por profesores y alumnos en la interacción educativa en que se concreta la transmisión cultural (Alonso Hinojal, I., 1980).

El salto de esté nivel del aula al nive! de la estructura social lo establece Bernstein aplicando esas herramientas sobre la configuración del conocimiento en ambos niveles, sobre la jerarquia de "status" y propiedad de esos conocimientos, la correspondiente de sus poseedores y el poder y control que, respectivamente, ostentan y ejercen sobre los demás. Es decir, el conocimiento está claramente estratificado en niveles superiores e inferiores, en el aula y la sociedad. Componentes fundamentales de esta estratificación son su prestigio diferencial; la jerarquia de saberes, por otra parte, se corresponde con la jerarquía social a través de la propiedad de unos y otros. La competitividad por los saberes y por el prestigio conduce a la restricción sobre los grupos menos poderosos y a la adscripción de diferentes contenidos curriculares a diferentes grupos sociales.

Bourdieu, al relacionar las prácticas pedagógicas y el curriculum con rasgos culturales propios de la sociedad francesa, 
se sitúa, por un lado, al borde del currículum "oculto" que trataré más adelante $y$, por otro, en el limite también entre los distintos capitales y sus respectivas formas de transmisión por él analizadas en trabajos posteriores (Bourdieu, 19 ). No sólo hay capitales sociales, culturales y propiamente económicos, sino que las clases privilegiadas en su dinámica de adaptación conservadora a los cambios sociales de todo tipo, utilizan hábiles estrategias de reconversión de partes de unos capitales a otros. Pues bien, la escuela, tanto por su curriculum como por su pedagogia, es el principal instrumento de la transmisión cultural, sin que sea ajena a las otras formas de transmisión.

Para estos autores pioneros, pioneros en estas nuevas reorientaciones, y para sus seguidores, el estudio del curriculum no sólo es el tema central del análisis de la educación por la sociologia, sino que, desde el estudio de ese tema central se va necesariamente pasando a otros relacionado con él hasta quedar cubierto todo el ambito educativo, pudiendo, y debiendo incluso, pasar al estudio de la estructura social mäs amplia. Es decir, la insatisfacción con el enfoque previo de la sociologia de la educación y especialmente con su concentración en el estudio macrosociológico de la desigualdad de oportunidades ha llevado a proponer (nuevas» direcciones alternativas y fundamentalmente el estudio del currículum. Podria, pues, hablarse de un movimiento en espiral: de una sociología de la educación muy focalizada a una sociología más abierta del conocimiento en general y del curriculum en particular; de aqui se ha ido ampliando el enfoque hasta incluir los temas más lejanos al punto de partida.

Este último aspecto, la expansión temática a partir del curriculum ha quedado ya apuntada en los autores citados y se seguirá comprobando en los que se citen en lo sucesivo con un motivo u otro. Por eso me ahorraré una enumeración más precisa, que juzgo innecesaria, para mostrar ahora algunos de los factores que impulsaron o hicieron posible ese proceso expansivo antes de entrar en el último tramo de la espiral: el político. Procederé por sucesivos contextos de amplitud creciente.

El primero se sitúa, sin duda, en la propia disciplina, la sociologia. El propio
Young se preguntaba, ¿por que no ha habido una sociologia del curriculum?, y se respondia que quiza por la propia organización del conocimiento en la misma sociologia, habituada a dar por supuesto el elenco de problemas que se le han ofrecido c incapaz de "construirse" otras alternativas (Young, M. F. D., 1970, pág. 40). Dentro de aquellos temas la limitación o, concentración en unos pocos ha sido evidente; concretamente en torno a los factores de desigualdad, de selección y reproducción. La aparición y expansión en sociologia de las perspectivas subjetivas descubrió nuevos temas de interés, como son, en general, los relativos a lo que ocurre en el aula y a lo que en ella se transmite y no se transmite.

Otros saberes cientificos también habian experimentado para esas fechas conmociones en sus seguras estructuras tradicionales. La filosofia y la historia de la ciencia se habian anticipado a la sociologia para relativizar los distintos saberes en razón de circunstancias exteriores, históricas o actuales.

En el propio ámbito educativo, $\underline{\mathbf{l a}}$ insatisfacción con los resultados ayudó a orientar el objetivo del análisis a lo que ocurre en el aula. La insatisfacción, primero, con las pretensiones de igualación social por la educación llevó al currículum y en varios paises a las pretensiones de homogeneizarlo; después, la sospecha de que aún así seguia habiendo mecanismos de desigualdad en el aula, más la creciente sensibilización hacia grupos especiales de culturas diferenciadas que presionó en el mismo sentido. Aún más recientemente, pero no lejos de lo anterior, la crisis del empleo y las preocupaciones por reorientar la educación hacia las necesidades del trabajo y de las empresas presionaron y siguen presionando. Hoy mismo se carga al pasivo del curriculum los problemas escolares más variados y preocupantes, como son la indisciplina y el aburrimiento.

Por supuesto, todos estos factores han actuado de distinta manera y con distinta fuerza en cada caso, como ha ocurrido especialmente con los factores políticos. Los dos que citaré en este ámbito tuvieron un papel destacado en Gran Bretaña, cuna de la renovación que analizamos. Me refiero a la tradicional lucha de base sindical contra los privilegios de la socie- 
dad tradicional y la gestión educativa descentralizada. Diversos documentos aparecidos. en ese pais a finales de los sesenta mostraban ya una dura y coincidente crítica, no. sólo a los resultados de la educación y a sus contenidos, sino también a la gestión realizada e incluso al escaso o nulo aporte de las ciencias encargadas de su estudio. La gravedad de la crítica se ponia de manifiesto desde el momento en que era lanzada tanto desde el lado derecho como el izquierdo del abanico político.

Como muestra significativa de lo que estoy diciendo transcribiré algunas de las más actuales cuestiones de política educativa que, a juicio de la OCDE, y recogiendo informaciones de los países miembros, presionan sobre los currícula y su planteamiento: las discontinuidades entre niveles educativos; estudios integrados o diferenciados en relación con la pretensión de igualar las oportunidades; opción entre objetivos cognitivos claramente señalados y metodologias informales; formación general o especializada a partir de ciertas edades, etcétera (Lauglo, J.).

\section{DE LA SOCIOLOGLA DEL CURRICULUM A LA POLITICA DEL CURRICULUM}

La sociología del curriculum, como núcleo de la nueva sociología de la educación, o de las nuevas direcciones en - sociologia de la educación, desde su misma aparición y acompañando su rápido crecimiento, fue progresivamente ampliando su temática y diversificando sus enfoques. Y esto en razón de múltiples factores: principalmente por la propia diversificación de la misma sociologia, y también por la polarización ideológica y política del medio, especialmente el medio educativo. Primero se van perfilando los distintos paradigmas en el enfoque del currículum, luego se van acentuando las diferencias y acumulándose las tensiones, hasta llegar a una especie de guerra de paradigmas y, finalmente, al cansancio de la propia guerra y a lo que hoy me parece una tregua o una guerra fria.

¿Cuáles son esos enfoques o paradigmas? No cabe esperar que haga aqui una síntesis imposible de los casi infinitos planteamientos y clasificaciones realizadas desde las distintas ciencias de la educa- ción. Pretendo simplemente un esquema minimo que permita desarrollar el hilo de la argumentación desde la perspectiva aqui aplicada, la de la sociologia. Me centraré en dos modelos polares y simplificados, de claras bases teóricas e ideológicas y de implicaciones sociales bien diferenciables, sobre los cuales, por otra parte, creo que confluyen en buena medida los demás. Uno será el modelo del curriculum tradicional, recibido, objetivo - curriculum como hechos, centrado en la concepción de unos saberes objetivos, bien definidos, si no plenamente, acumulativos, ordenados en disciplinas, agrupados en facultades y atribuidos a bien. diferenciados especialistas. Esos saberes, materia misma de los curricula, pueden y suelen tener muy distinta consideración, como ponen de manifiesto calificativos del tipo de superiores e inferiores, puros y aplicados. Hay entre esos saberes y sus disciplinas una clara, jerarquía que, lógicamente, se transmite a sus cultivadores y a los centros correspondientes.

La denominación de curriculum recibido, usada por Eggleston (1977) supone alusiones interesantes. Se refiere a que es un curriculum recibido por profesores $y$ alumnos, a los que no les queda otro cometido que su asimilación lo más fiel y rigurosa posible como parte de un orden superior también dado. Quienes conciben así el currículum están preocupados, sobre todo, por los objetivos de la enseñanza, por el orden, la asimilación, la competencia resultante y su medida. De larga tradición, este modelo es, según algunos, el intento de aplicar a la enseñanza el taylorismo industrial que incluiría, como criterio definitivo para juzgar la bondad de la escuela; la medida de los conocimientos asimilados por los alumnos y su comparación con el coste de haberlos obrenido. El esquema es el bien conocido de Tyler, quien incluye como lógicas etapas de la planificación del curriculum la fijación de objetivos, los contenidos, la metodologia y la evaluación.

El modelo fundamentalmente alternativo, el subjetivo, reflexivo o centrado en el alumno, no se orienta tanto a la adquisición de conocimientos, como al futuro funcionamiento social de los perceptores. Es, más que subjetivo, situacional y ecléctico en cuanto no prescinde en absoluto de los conocimientos objetivos, sino que los relativiza y subordina a las experien- 
cias y propósitos del alumno: La relativización de los conocimientos es correlativa a la relativización del curriculum, para hacerlos corresponder a las diferentes situaciones sociales. En este modelo, las etapas del desarrollo curricular varian consecuentemente: la primera sería el análisis situacional, seguiria la fijación de objetivos, la programación, interpretación, valoración y retrocorrección. En todo caso, las etapas aqui tendrian un sentido muy distinto y mucho más flexible.

La constelación de modelos o paradigmas que se sitúan o integran en torno a estos polares podrian especificarse prestando atención al elemento o elementos que destacan en sus respectivos procesos de desarrollo. Asi, por ejemplo, en el primer tipo podrian diferenciarse modelos centrados en los conocimientos científicos mismos, en los objetivos de la enseñanza, en la evaluación de los conocimientos adquiridos o en los mecanismos y tecnologia de la transmisión. En torno al segundo tipo cabe también insistir en el alumno como individuo y sus necesidades, en el contexto social en el que vive o al que se le pretende incorporar, en el proceso o interacción en que la enseñanza consiste, etc.

En España, un reciente trabajo de un pedagogo de orientación sociológica (Gimeno Sacristán, J., 1982) se situaría en esta interpretación. Al estudiar la pedagogia por objetivos, comenta y critica el primer tipo de curriculum, el centrado sobre los objetivos, al que llama curriculum tecnocrático, para contraponerlo a otro centrado sobre el proceso, que se podria denominar humanista, y cuyo estudio deja para otra ocasión. A la vez ofrece este autor una tipología de enfoques, del currículum que yo me atreveria a reunir también en torno a los dos tipos ideales anteriores: sus tipos tecnológico y racionalista se incluirian en mi primer grupo, y el psicológico y social, en el segundo.

Aunque sea inútil, advertiré que estoy forzando lo ya forzado, es decir, clasificaciones que, como todas, implican simplificaciones de la realidad. Por otra parte, los supuestos y exigencias de los dos tipos extremos en que se han condensado los diversos enfoques del curriculum difieren mucho en su grado de concreción, análisis y aplicación. Los del segundo tipo gozan hoy de «buena prensa» y están muy difundidos y aceptados a nivel de principios y orientaciones generales, pero mucho menos en su aplicación práctica y consecuente.

Pero a lo que queria llegar es a la multitud y diversidad de implicaciones de unas y otras concepciones. Una vez más me limitaré a simples alusiones, siguiendo contextos sucesivos y relacionados entre si. Cada uno implica, desde luego, una diferente concepción de lo que es el saber, el saber cientifico más concretamente, como algo riguroso, seguro y bien delimitado, o, por el contrario, como un producto social variable en cada caso no sólo en su cantidad, sino también en su calidad, orientación, distribución, uso, etc.; junto a esta concepción estaría la diferenciación y jerarquización de aquellos que deben transmitirse a quiénes, cuándo y cómo. Supone tambiên una concepción distinta de los roles educativos, del profesor, del alumno y dé los más intervinientes, con diferentes grados de definición, rigidez, posibilidades de expresión, de autonomia, etc. Implican, por tanto, distintas concepciones de lo que sea la educación y para qué, y de cómo deba desarrollarse la actividad educativa e incluso la organización del-aulá, los centros y el sistema escolar mismo. Por encima, o por debajo, de todo ello, están lógicamente implícitas concepciones diferentes sobre el individuo, la sociedad y sus relaciones.

Todas estas y otras conexiones e implicaciones están siendo puestas de manifiesto y analizadas por los sociólogos que se ocupan del curriculum o sencillamente de la educación. Con todas sus limitaciones, me atrevo a citar a Basil Bernstein como ejemplo de analista que, centrado en el conocimiento escolar y su transmisión, ha tratado de relacionar criticamente este núcleo con casi todos los citados y, desde luego, con la estructura social de poder y los mecanismos de control (Bernstein, B., cap. s). Su trabajo es un ejemplo de análisis interniveles $e$ interenfoques (Alonso Hinojal, I., 1980-b).

Es frecuente establecer otras conexiones con el currículum, una de ellas relativamente frecuente que no comparto. Me refiero al supuesto apoyo o fundamentación de los principales enfoques en sen- 
das disciplinas entre las ciencias de la cducación. Daré dos ejemplos. Eggleston considera que la perspectiva llamada por él "recibida", se apoya y recibe su soporte teórico de la filosofia y de la psicologia, mientras que la perspectiva alternativa, la reflexiva, se apoya en la sociologia. Es muy tajante al respecto: «Si la perspectiva recibida obtiene su fuerza del soporte que le prestan filósofos y psicólogos, los que la desafian han recibido el sostén, con casi igual regularidad, de los sociólogos". (Eggleston, J., 1977, p. . 64). El propio Gimeno Sacristán (1982) hace parecida, aunque más matizada, asignación, considerando de manera más o menos contundente que la perspectiva racionalista se apoya en la filosofia y la perspectiva «social» en el enfoque sociológico. En cambio, el «modelo de objetivos tiene detrás de si una fundamentación psicológiča determinadan:

Desde la sociología, que es mi parcela, no se puede mantener esa adscripción tan clara y contundente en apoyo de uno de los enfoques curriculares y sospecho que tampoco desde otras parcelas, hasta donde llegan mis nociones de ellas. Significaria negar el pluralismo o pluralidad de enfoques que hace tiempo están aceptados. Considero más aceptable considerar que detrás de esos dos enfoques tipo del curriculum están sendas posiciones sociológicas que, continuando con las simplificaciones, corresponderían a dos posiciones epistemológicas: la interpretación objetiva y la subjetiva del conocimiento sociológico. Y si se quiere una tipologia más diversificada de los enfoques curriculares, también se pueden aportar clarificaciones o paradigmas de la sociología actual que podrian hacerse corresponder con aquéllos. Asi lo hice en otra ocasión y referido a la sociologia de la educación (Alonso Hinojal, I., I $9^{80-a}$, cap. 4), añadiendo a la dimensión epistemológica, antes citada, la ontológica, según que prevalezca una visión consensual o estática de la realidad social u otra conflictiva o cambiante. La tipologia asi resultante es mucho más completa y nos daria varios enfoques teórico-ideológicos del curriculum, como de cualquier otra parcela social: a) el objetivo consensual; b) el objetivo conflictivo; c) el subjetivo consensual, y d) el subjetivo conflictivo. Esta $I 24$ : mucho más rica.y matizada para el análi- sis del curriculum. Ya que no procede realizarlo aqui, añadiré, sin más, unos ejemplos como testimonio hipotético de su adecuación metodológịca.

En el primer grupo caerian los autores más numerosos y comúnmente considerados como funcionalistas y apoyados en el concepto tradicional de socialización, desde Parsons a Banks. Enfoque predominante y con frecuencia muy elaborado, como el ofrecido por P. W. Musgrave (1971), cuya definición inclui al comienzo, que tiene en cuenta dos niveles (macro y micro) y tres principales elementos: objetivos, agentes y : contenido. En el paradigma objetivo conflictivo se situarian, entre otros, autores que han adoptado el principio de correspondencia, como Bowles y Gintis, el de la reproducción como Bourdieu y seguidores, o el de las redes como Baudelot y Establet. Del enfoque consensual subjetivo sería un ejemplo la defínición de currículum de Mac Donald: «una construcción social en la que la selección y la organización del conocimiento... es una elección entre posibles alternativas» o la más breve $y$ radical de Maxime Greene, "una posibilidad para el alumno como persona interesada ante todo en dar sentido a su propio mundo" (Greene, 1971).

Como es evidente, la distinción ontológica lleva muy directamente a posiciones ideológicas y políticas muy diferenciadas o, mejor, contrapuestas y que en este sentido intentan sobrepasar e incluso absorber la distinción epistemológica. Es el caso de algunos autores ya citados. Whitty, por ejemplo, después de criticar duramente la posición dominante, la del currículum como mercancía, y aceptä con reparos la posición alternativa, la del currículum como práctica, ya que reconoce a profesores y alumnos unas posibilidades de cambio puramente teóricas, propugna una lucha de profesores y alumnos por el cambio uno independiente de la de trabajadores y comunidades», ya que los obstáculos al cambio están más allá del aula y los conocimientos (Young, M., 1977, p. 248).

El conflicto en torno al curriculum es hoy algo totalmente reconocido a nivel teórico y en la práctica, en estado latente o manifiesto y más o menos institucionalizado según los países. Las frecuentes referencias a su carácter confidencial y. 
reservado, como las expresiones inglesas de "secret garden", "secret service", "black box"s, etc., hacen referencia a situaciones pasadas que han sido superadas, al menos en las discusiones teóricas, en los paises más avanzados. En otros, la discusión apenas se ha iniciado, pero progresara si las condiciones politicas, en concreto democráticas y participativas, le son favorables.

Pero el curriculum es algo tan central en la educación y tan relevante en la dinámica social y cultural, que las tensiones o los conflictos se manifiestan en muchos frentes. He aludido al teórico, alli donde las teorias educativas, desde unas y otras ciencias, están suficientemente desarrolladas $y$, por tanto, diversificadas. En nuestro país la calma, o retraso, en este último terreno se verá pronto rota y sobrepasada por la discusión animada. Pero hay también conflicto latente o manifiesto entre grupos profesionales, actuantes como grupos de presión, conflictos no independientes de los.anteriores. Tomemos como ejemplo un país, el Reino Unido (como ya indiqué, el caso de España merecerá consideración aparte) donde la cuestión es más tratada. Están, ante todo, los maestros (los cultivadores del (garden»), que «tienen toda la razón en mostrarse cautelosos respecto de influencias externas..., pero que se equivocarían en reclamar que sólo ellos tienen derecho a discutir el curriculum» (Lawton, 1980, p. 134). Especialmente abusivo sería el atribuir ese derecho a maestros o centros individuales. Diferencia Lawton cinco niveles de decisión en temas curriculares referidos a la organización administrativa de su país, aplicables en buena medida al nuestro con las transferencias educativas en marcha: nivel nacional, regional y local, institucional (centro), departamental e individual. Se reconoce que a los profesores corresponde, evidentemente, el control último, puesto que ellos son los que toman las decisiones frente al alumno, pero no deben prescindir de los otros niveles de decisión, a los que debe exigirseles paralela consideración. «En una sociedad democrática, dirá Lawton, no sólo es importante contar con un currículum más valioso; es también importante que los mecanismos de su desarrollo y cambio estén adecuadamente controlados y que ese mecanismo se vea como transparente y apropiado por todos los que tienen interés legítimo en él. Probablemente sean inevitables disputas ocasionales sobre el control del curriculum, pero es hora de que las reglas de juego sean públicas» (Lawton, 1980, p. 1 39).

La sociologia, que tiene el conflicto como uno de sus temas clásicos de estudio, está claro que debe prestar atención al curriculum, puesto que es un foco real o potencial de conflicto múltiple. En caso que éste aún sea latente o potencial, es decir, de que aún se cultive el usecret garden», el contribuir a romper el secreto seria también un cometido propio. El "confidencialismo» dice mucho del carácter abierto o cerrado de una sociedad, de los intereses que reclaman protección y de los grupos que tienen poder para proteger sus intereses. En la administración pública, el secreto es a veces utilizado para fines que tienen muy poco que ver con los intereses de los ciudadanos» (Oyen, 1982).

El tránsito de una sociedad y una educación jerárquicas a otras democráticas y participativas debe romper secretos y. poner de manifiesto demandas y exigencias diversas y a veces contradictorias; es decir, poner de manifiesto conflictos. y forzar a su pronta institucionalización y al establecimiento de unos mecanismos de control acordes con las fuerzas sociales que pueden y deber entrar en funcionamiento para orientar los inmimnentes cambios en los planes de estudios que urgen esas mismas fuerzas del cambio. Tenemos ejemplos bien recientes e ilustrativos al respecto, como son las nuevas exigencias que al sistema educativo transmite la actual crisis profesional y de empleo. Si bien esas exigencias son generales, mientras en unos paises como los de la Europa Occidental se discuten las implicaciones desde la derecha y deside la izquierda, en los paises más al este se trasladan e imponen sin mas hasta en los niveles educativos más bajos.

\section{s. EL NIVEL TIPICAMENTE SOCIOLOGICO: EL OCUL.TO}

Hasta aquí he tratado de mostrar la pertinencia del análisis sociológico del curriculum y cómo de alguna manera se ha hecho en el pasado y se hace hoy en sus aspectos más destacados y visibles. En lo que resta voy a insistir en lo 
mismo, pero mostrando cómo hay aspectos del curriculum, y de la educación, que, por menos manifiestos, e incluso por ocultos, no dejan de ser menos importantes y constituyen materia tipica del análisis sociológico, cualquiera que sea la perspectiva téórica desde la que se actúe. Si en el punto anterior se llamó la atención respecto del "secreto sobre el curriculum" y su interés y adecuación para el análisis sociológico, aquí me referiré al «curriculum secreton y su naturaleza central para dicho análisis. Porque hay un curriculum oculto o latente constituido por el conjunto de valores, normas y prácticas que son «enseñadas implícita pero efectivamente en las escuelas y de las que los profesores no hablan en sus declaraciones sobre fines y objetivos» (Apple, 1979, p. 84). Es aquello que se aprende en la escuela, además de lo que ésta propone enseñar y a veces contra lo que se intenta enseñat. Es aquello sin lo cual el propio funcionamiento de la escuela sería imposible en cuanto que las normas y procedimientos preestablecidos no pueden ser completos $y$ deben ser rellenadas por pautas y normas «improvisadas». Su carácter supletorio, sin embargo, no significa importancia secundaria, pues no sólo su contenido es indispensable para la realización del curriculum oficial o manifiesto, sino que, en caso de contraposición entre uno y otro, puede no sólo anular, sino hasta imponerse a éste.

A título ilustrativo citaré algunos de los componentes considerados como típicos del currículum oculto (Jackson, $\mathrm{P}$. W., r968). Enseña a vivir «en multitud» controlando y regulando los deseos de hablar con los compañeros o con el profesor; enseña la fundamental virtud de la paciencia, necesaria para aprovechar y para perder el tiempo; a aceptar los juicios y valoraciones de los demás, profesores y alumnos; a competir y los limites en que puede hacerse; a cómo vivir en una sociedad que constantemente evalúa y jerarquiza; a colaborar en la regulación de las actividades escolares, aceptando o alterando la que impone el centro y el profesor; todo ello acatando o elaborando ciertos códigos y sus sanciones.

El curriculum oculto se incorpora en buena medida antes ya de llegar al centro parte de la cultura social relativa a la educación, lo que facilita la incorporación $y$ el trasvase de unos centros a otros, es decir, la integración en los grupos de clase. No es, pues, algo encapsulado $y$ que aisla, más bien conecta con la cultura general, con la que cambia y a la que en alguna medida influye.

En cualquier caso, la defínición o delimitación del llamado currículum oculto no es fácil y resulta más asequible por la vía negativa y residual. Tampoco es fácil responder al porqué se da y de dónde procede, ya que se trata de un fenómeno social muy global que se produce en la convivencia de todo grupo estable sometido a unos objetivos y normas preestablecidas, es decir, a una organización formal. Surge precisamente para adaptarse, completar o defenderse de las exigencias de ese tipo de organización. Supone, pues, un poder de oposición o negociación con la organización y, en consecuencia, implica una redistribución del poder; formalmente ubicado por la organización, en favor, en principio, de quienes deben someterse a él, en nuestro caso, en favor de los alumnos.

No puede decirse, ni mucho menos; que la sociologia de la educación, en los paises en que está desarrollada, no se haya ocupado del curriculum oculto desde que Jackson acuñara la expresión en 1968. Lo está haciendo cada vez con mayor atención, precisamene desde que se diversificaron las posiciones teóricas y ganaron fuerza los enfoques criticos, dentro del creciente clima de decepción o escepticismo respecto de la educación y sus logros esperados normalmente de la organización y el currículum oficial y manifiesto. Aqui no voy, lógicamente, a pasar revista a esos trabajos, sino simplèmente a continuar y concluir la argumentación hasta aquí desarrollada, mostrando cómo en el llamado curriculum oculto la pertinencia del análisis sociológico es aún más clara y prometedora, aunque nada fäcil. Trataré de mostrar también cómo ese curriculum oculto es especialmente decisivo para ciertos fines o contenidos de la educación.

Para intentarlo lo más brevemente posible me valdré de un reciente intento de clarificar las diversas definiciones del curriculum oculto, hecho desde la filosofía de la educación, pero reconociendo que en este tema la "argumentación es primariamente sociológicas. (Gordon, D., 1982). Para Gordon predominan tres ti- 
pos de definiciones en razón de que se centren, preferentemente en los resultados, en el clima o ambiente escolar y en los modos de influencia sobre el alumno, en todos los casos latentes o encubiertos. Sin entrar en los detalles de la tipologia, el mismo autor añade otra caracterización del curriculum oculto: la de ser congruente y comprensivo, es decir, el implicar todos los aspectos de la vida escolar y el seguir una línea temporal homogénea y persistente.

Pienso que, más que tipos de definiciones diferentes, la anterior especificación destaca elementos importantes a incluir en una definición más comprensiva, en la que podrian ir incluidos aspectos muy dispares señalados por numerosos autores. Ejemplos de lo último podrían ser la inclusión o caracterización del curriculum oculto como la forma de desarrollar el manifiesto (Barton, et. al., 1980, págs. 163 y ss.), la manera de presentar las ciencias físico-naturales y el implicito reconocimiento o rechazo del conflicto social (Apple, 1979), o los códigos pedagógicos de Bernstein, apoyados en la relación entre contenidos y entre transmisores y receptores (Bernstein, B., 1977, cap. s).

Por otra parte, creo que, a los múltiples intentos de definición y caracterización de lo que hoy se considera curriculum oculto, habria que añadir descubrimientos varios de otros aspectos del proceso educativo también ocultos y también importantes en sus consecuencias. Es el caso de la pedagogía invisible que el propio Bernstein descubre, junto a la visible y clásica, relacionada con la aparición de las nuevas clases medias y caracterizada como aquella pedagogia en la que el control es indirecto, la autonomia del niño aparentemente muy grande, las evaluaciones múltiples y con criterios difusos, etc. (Bernstein, B., 1977, cap. 6). Es el caso también de la pedagogia oculta a que se refiere Descombe cuando afirma que, a pesar de la intensidad y diversidad de entrenamiento a que se somete hoy a los profesores y a pesar de la diversidad de situaciones en las que luego ejercen, todos tienden a compartir y mantener intocables un par de principios: el de la no interferencia de otros profesores de la propia clase, o principio de la privaticidad, y el del mantenimiento del orden en la clase a toda costa y por el propio profesor (Descombe, M., 1982).

Como se ve, hay toda una multitud de aspectos poco o nada visibles en la educación que, sin embargo, producen efectos importantes o definitivos en el funcionamiento y resultados de las instituciones educativas. Por otra prte, esos resultados no buscados y a veces no detectados se refieren especialmente a cuestiones importantes del conocimiento y comportamiento social más general que el supuestamente educativo. Se refieren a cuestiones como la concepción de lä propia sociedad y la cultura, a las instituciones básicas y su funcionamiento, a las reglas del juego político, a los estereotipos y actitudes hacia ciertos grupos o categorias sociales, etc. Hay sistemas escolares en los que este tipo de temas o alguno de ellos se incorporan al curriculum explícito mientras que en otros, no. Lo curioso es que no siempre obtienen unos resultados finales mejores los que los han incorporado, y que incluso en algunos se obrienen resultados opuestos a los buscados.. En nuestro país, un buen ejemplo del pasado reciente seria la uformación política» $\mathrm{y}$, si no se presta atención a los factores ocultos, podría serlo en el futuro inmediato la enseñanza de la Constitución. El caso de la educación politica es quizá el paradigmático y mejor estudiado, incluso en nuestro país, donde también se ha comprobado cómo conocimientos, actitudes y comportamientos no siempre varian paralelamente (Fuente, C., y Muñoz-Repiso, M., 198I). Algo parecido, aunque menos frecuente y estudiado es el caso de la utilización expresa de la educación contra la discriminación social por sexos. Alli donde se han introducido contenidos expresos en el curriculum, sólo se han conseguido resultados positivos cuando se han_visto reforzados por el curriculum oculto; en palabras de Sara Delamon, usólo con profesores comprometidos en el cambio de las relaciones sociales entre los sexos es posible el cambio de actitudes en los alumnos, y aún entones sólo si son hábiles en el manejo de los materiales didácticos» (Delamont, S., 1980, pág. 112).

Pero estas cuestiones más concretas y referidas a nuestro pais deben esperar el tratamiento que se merecen y que no es posible aqui, donde sólo me resta mostrar algunas lagunas y posibilidades de la sociología aplicada a la educación. 
Es de notar ante todo el hecho sorprendente de que la sociologia y otras disciplinas estudiosas de la educación hayan ido lentamente descubriendo estos aspectos secretos $\mathrm{u}$ ocultos y denominándolos de formas tan llamativas («jardin secreto", "caja negra", "pedagogia "oculta", "pedagogia invisible", "pacto negro", etc.), sin que la sociologia, a quien las otras teorias incluso han atribuido especialmente estos ámbitos, se haya ocupado preferentemente y sistemáticamente de ellas, siendo así que se les reconoce tanta trascendencia social. Para mi es aún más sorprendente cuando, por un lado y como dije antes; esos niveles y ámbitos han sido tradicionalmente considerados como los más propios y prometedores de la sociología y cuando, por otro, en ella existen desde antiguo instrumentos conceptuales para afrontar este tipo de cuestiones. A modo de ejemplo y sugerencia para posteriores elaboraciones me referiré a algunas de estas posibilidades.

En el meollo de la perspectiva sociológica -está la «percepción de la dinámica autónoma y con frecuencia oculta de las entidades humanas colectivas" a que se refería hace ya bastantes años Robert $\mathrm{K}$. Merton cuando distinguia y elaboraba las funciones sociales manifiestas y las latentes, diferenciando los objetivos sociales y públicamente definidos de una institución, de los subyacentes, no percibidos pero objetivos de la misma (Merton, R. K., 19 , pág. 62). Pot funciones manifiestas entiende raquellas consecuenicias objetivas para una determinada unidad (persona, grupo, sistema social o cultural) que contribuyen a su ajuste o adaptación y que intenta que sirvan para eson; las latentes, en cambio, son «las consecuenias no intencionadas ni reconocidas". Los propósitos hermenéuticos de la distinción tratan, ante todo, de clarificar las acciones sociales aparentemente irracionales $o$ inexplicables $y$ la frecuente atribución de tales comportamientos a causas o condicionamientos extraños y a veces ajenos al medio social. Pues bien, esta distinción, que Merton elaboró y tomó del psicoanálisis, ha sido utilizada de un modo u otro por los más destacados pensadores sociales, como el propio Merton ha mostrado, desde Durkheim y Summer a Mead y Mclver. $Y$ es una distinción que en pocos 128 ámbitos puede ser tan adecuada y útil mostrarlo en relación con el curriculum, pero piénsese lo que ocurre a otros niveles entre las pretensiones explicitas y los logros reales de la educación.

Desde uno de los campos más viejos y cultivados de la sociologia, la teoria de las organizaciones, es tipica la distinción y el análisis en ella apoyado, entre organización formal e informal. También las organizaciones educativas han sido consideradas y estudiadas como organizaciones formales, pero con escasa atención a las correspondientes y siempre presentes organizaciones informales y sus consecuencias. En el ámbito industrial, especialmente, se han analizado ampliamente sus componentes (valores, normas, sanciones, estructura jerárquica, liderazgos, etc.) y sus consecuencias para los individuos: el grupo, la organización formal y la sociedad misma. En lo apuntado sobre el curriculum manifiesto y sobre todo el oculto, creo que he puesto de manifiesto la conveniencia de un tratamiento de las organizaciones educativas desde esta doble e intimamene relacionada perspectiva.

Es muy posible que también se haya contribuido a lo que estoy destacando desde la propia investigación educativa, que, al multiplicarse cuando se multiplican los recursos, lleva a veces a la trivialización y demostración de lo evidente mediante un "método" paralelamente generalizado como es ula encuesta cerrada para ordenador".

Si estas carencias, al menos relativas, son ciertas cabe preguntarse a qué se deben. Sin mayores pretensiones, creo que se pueden hacer algunas sugerencias interpretativas, como son: el relativo desprestigio inicial de la sociologia de la educación o sociología educativa; su correlativo aislamiento, también relativo y variable, respecto de la sociologia general y sus especialidades más sólidas; en tercer lugar, estarian las causas apuntadas por los unuevos» sociólogos de la educación y ofrecidas como críticas a la "vieja" sociología de la educación, criticas que algunos de ellos han hectio a los demás y no han aplicado a su propio trabajo. Aqui podriamos considerar como ejemplo la incomunicación entre dos conceptualizaciones y análisis correspondiente de la misma realidad: la acción incorporadora de la sociedad sobre sus nuevos miembros. En efecto, los conceptos de educa- 
ción y socialización han sido utilizados aisladamente por sendas disciplinas y aplicados también separadamente a ámbitos concretos del mismo proceso. El primer concepto y su consiguiente enfoque apenas ha ido más allá del estudio de la educación formal; el segundo y su interés por los aspectos informales y latentes casi no ha entrado en el campo de la educación, permaneciendo en el análisis de la familia, la comunidad y otros medios sociales. La discontinuidad y rigidez en la aplicación de esta doble conceptualización, característica de una fuerte «clasificación" en la terminologia de Bernstein, que aqui he puesto minimamente del manfiesto, ha sido ampliamente denuncia$\mathrm{da}$ en otros lugares $y$ por diversos autores. Dirá Leichter: "La dicotomia conceptual sobre la existencia del niño entre educación y socialización, la una característica de la escuela y la orra de la familia, parecen haber sido reificadas como fenómenos reales. La consecuencia hasta hace poco parece haber sido el fracaso en considerar de un lado el proceso educativo de la familia $y$, de otro, el de socialización en la escuelan (Leichter, 1974, pág. 44).

\section{Resumen}

En contra de ciertas afirmaciones, se muestra que siempre que la Sociologia, se ha ocupado realmente por la educación ha prestado ateneión a los contenidos, especialmente en épocas de pluralismo y tensión teórica e ideológica, como es la actual. Se destaca el "currículum oculto" como ámbito especialmente adecuado para el análisis sociológico.

\section{Summary}

Against certain affirmations, it is possible to point out that when Sociology has really dealt for education, it was taking in consideration the contents, specially on times of pluralism and theoretical $\rightarrow$ s well as ideological-tension, as it happens in our period. One should emphasize the "hidden curriculum" as a field mostly appropriate for the sociological analysis.

\section{Résumé}

A l'encontre de certaines affimations, il est possible de montrer que lorsque la Sociologie s'est réellement occupé de l'éducation, elle a toujours prété attention aux contenus, surtout dans les moments de pluralisme et de tension théorique ainsi qu'idéologique, comme c'est le cas actuellement. Il est possible de souligner le kcurriculum occulte» en tant que cadre spécialement adéquat pour l'analyse sociologique.

\section{Referencias}

Alonso Hinojal., I. (1980-2): Educación y Sociedad. Las sociologias de la Educación. Madrid, C. I. S.

Alonso Hinojal., I. (1980-b): «Bernstein en la encrucijada de la Sociología de la educacións. Revisto Española de Investigaciones Sociológicas, núm. 11.

Alvaro, M.; Dendaluce, I.; MuÑoz-Repiso, M., y Perez, G. (1981): Los estudiantes esparioles y los valores democraticos. Madrid, M. E. C.

APple, M. (1979): Ideology and Curriculum. London, Routledge and Kegan Paul.

Barton, L.; Meigham, R., y Walker, S. (Eds.) (1980): Scbooling, Ideology and Carriculum. Barcombe, England, The Falmer Press.

Bernstein, B. (1977): Class, Codes and Control, vol. 3. London, Routledge and Kegan Paul.

Delamont, S. (1980): Sex Roles and the Scbools. London, Rourledge and Kegan Paul.

Descombe, M. (1982): «Hidden Pedagogy and its implications for Teacher trainingn. Britisb Journal of Sociology of Education, vol. 3, núm. 3, págs. 249 y ss.

Durkheim, E. (1938): L'evolution pedagogique en France. Paris, P. U. F.

EGGl.eston, J. (1977): The Sociology of School Curriculum. London, Routledge and Kegan Paul.

ESL.AND, G. M. (1971): «Teaching and Learning as the Organization of Knowledgen. En Young M. F. D., Knowledge and Control. Londres, MicMillan.

FuENTE, C., y MUÑoz-RePiso, M. (1981): Preparación para la vida en una sociedad democrática en las escuelas de la Europa Meridional. Madrid, M. E. C. 


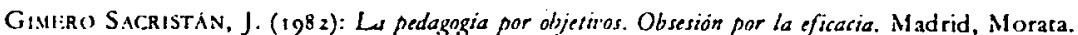

-Gordon, D. (1982): "The Concepts of Hidden Curriculum". Journal of Phiiosophy of Education. vol. 16. número 2.

GrEenE, M. (1971): “Curriculum and conciousncss". 7he Record. vol. 3, num. 2.

JACkson, P. W. (1968): Life in Classroom. Nueva York, Holt, Rinehart and Winston.

LAug1.0. J. (1979): «Policies for basic education. Some current curriculum issues». Paris, OCDE, $S M E / E T / 1979.44$.

LAwiton, D. (1980): The Policies of sbe Scbool curriculum. Londres, Routledge and Kegan Paul.

LAWTON, D. (1981): uThe curriculum and the curriculum change". En Simon, B., y Taylor, W'. (Eds.). Education in the Eighties. Londres, Batsford.

Leichter, H. J. (Ed.) (1974): Tbe Family as Educator. Nueva York Teacher College Press.

Mannheim, K. (1966): Introducción a la Sociologia de la Educacion. Madrid, Ed. de la Revista de Derecho Privado.

MERTON, R. K. (I957): Social Tbeory ans Social Structure. Nueva York, Free Press.

Musgrave, P. W. (1973): Knowledge; Curriculum and Change. Melbourne, Angus y Robertson.

Or.IVER, A. I. (1965): Curriculum Improvement. Nueva Yok, Dodd Mead.

OYen, E. (1982): "Confidentiality: Theory and Practicen. Current Sociology, vol. 30, núm. 2, pags. t-g.

YOUNG, M. F. D. (1971): "An Approach to the Study of Curriculum as Socially Organized Knowledge". En Young, M. F. D. (1971). Knowledge and Control. Londres, McMillan. 